\title{
Thermionic emission from small clusters: direct observation of the kinetic energy distribution of the electrons
}

\author{
H. Weidele ${ }^{\text {a }}$, D. Kreisle ${ }^{\text {a }}$, E. Recknagel ${ }^{\text {a }}$, G. Schulze Icking-Konert ${ }^{\text {b }}$, \\ H. Handschuh ${ }^{\mathrm{b}}$, G. Ganteför ${ }^{\mathrm{b}}$, W. Eberhardt ${ }^{\mathrm{b}}$ \\ "Unicersität Konstanz, Fakultät für Physik, D-78434 Konstanz, Germany \\ ${ }^{\mathrm{b}}$ Institut für Festkörperforschung, Forschungszentrum Jülich, D-52425 Jülich, Germany
}

\begin{abstract}
Photoelectron spectra of small mass-separated $\mathrm{W}_{n}^{-}$clusters cooled in a seeding gas are recorded with a low laser flux that ensures single-photon events. The spectra exhibit (i) sharp features due to direct photoemission and (ii) a broad emission signal whose intensity decreases with increasing kinetic energy. The latter is caused by delayed electron emission, a process similar to bulk 'thermionic emission'. The kinetic energy distribution of these electrons can be approximated by a Boltzmann function with a temperature corresponding to the photon energy divided by the number of vibrational degrees of freedom of the cluster.
\end{abstract}

\section{Introduction}

Thermionic emission of electrons from hot metal surfaces is a well-studied phenomenon in solid state physics [1], and the distribution of the kinetic energies $E_{\text {kin }}$ of the electrons emitted from a surface can be described approximately by a Boltzmann distribution $\exp \left(-E_{\mathrm{kin}} / k_{\mathrm{B}} T\right)$ [2], with $T$ denoting the temperature of the solid. Delayed ionization following multiphoton absorption in strong laser beams has been observed in atoms [3], molecules [4-6] and small metal clusters [5,7-9], and can be attributed to electronic or vibronic autoionization (autodetachment in the case of negative ions) or to thermionic emission. In an autoionization process discrete electronic and vibronic levels are populated to store the energy of the absorbed photon(s) and, following some decay mechanism, an electron with a defined kinetic energy is emitted. In contrast, electrons resulting from thermionic emission exhibit a quasicontinuous energy distribution. For neutral refractory metal clusters (e.g., $\mathrm{W}, \mathrm{Nb}$ and $\mathrm{Ta}$ ) this process has been studied in detail by measuring the time constants of the delayed ionization following multiphoton excitation [7-9]. Delayed electron emission has also been found for $\mathrm{W}_{n}^{-}$cluster anions excited by multiphoton absorption [10]. Similar experiments have focused on the mechanism of delayed emission of $\mathrm{C}_{60}$ and $\mathrm{C}_{60}^{-}$[11-16]. We examine here the transition from a non-thermal energy spectrum, characteristic of molecules, to a spectrum characteristic of thermionic emission.

Most of the above experiments [7-16] exhibit broad but discrete distributions of the internal energies of the 'superheated' clusters, since the number of absorbed photons in multiphoton processes is not 
exactly defined (Poisson distribution). Accordingly, the time dependence of the electron emission is a superposition of several exponential functions. Furthermore, most experiments study only the time dependence, which alone does not suffice to distinguish between electronic autoionization and thermionic emission, for example, since both processes lead to an exponential decay. More information is available from a measurement of the kinetic energy distributions, but we are aware of only one experiment in which the kinetic energy of the emitted electrons has been determined [16]. However, in this experiment multiphoton excitation was used to generate the 'superheated' clusters.

We study here the kinetic energy distributions of the electrons emitted from clusters with a well-defined size and internal energy [17]. Negatively charged clusters are prepared near room temperature and are mass-separated. The absorption of a single photon with an energy $h \nu$ larger than the electron affinity results either in direct photoemission according to

$$
\begin{aligned}
\mathrm{M}_{n}^{-}+h \nu & \rightarrow \mathrm{M}_{n}+\mathrm{e}^{-}, \\
& \rightarrow \mathrm{M}_{n}^{*}+\mathrm{e}^{-}, \\
& \rightarrow \ldots,
\end{aligned}
$$

or in a fast thermalization of the energy and a subsequent delayed emission according to

$$
\begin{aligned}
\mathrm{M}_{n}^{-}+h \nu & \rightarrow\left(\mathrm{M}_{n}^{-*}\right)_{E_{1}} \\
& \rightarrow\left(\mathrm{M}_{n}^{*}\right)_{E_{2}}+\mathrm{e}^{-} .
\end{aligned}
$$

In both processes the initial state is the electronic ground state of the cluster anion $\mathbf{M}_{n}^{-}$. In process (1) the final states are the electronic ground and excited states of the neutral cluster $\mathbf{M}_{n}, \mathbf{M}_{n}^{*}$. The spectrum of the kinetic energies of the emitted electrons exhibits distinct features corresponding to allowed transitions. In process (2) the absorption of the photon results in a 'hot' (internal energy $E_{1}$ ) cluster anion, which subsequently cools by electron emission resulting in a neutral cluster with lower internal energy $E_{2}$. The spectrum of the kinetic energies of the electrons is a smooth exponential distribution corresponding to a 'temperature' $T=E_{1} / k_{\mathrm{B}}(3 n-6)\left(k_{\mathrm{B}}\right.$ $=$ Boltzmann constant, $n=$ number of atoms). All the 'superheated' clusters $M_{n}^{-*}$ so prepared have approximately the same internal energy, which is similar to the energy of the absorbed photon. As long as the photon energy is much larger, the initial thermal energy can be neglected.

The recorded photoelectron spectra exhibit relatively sharp peaks assigned to direct photoemission and a smooth emission signal that increases monotonically with decreasing kinetic energy. The kinetic energy distributions of these 'thermionic' electrons are fitted by a Boltzmann distribution. In addition, the vertical detachment energy ( $\approx$ electron affinity) is extracted from the spectra and compared to the estimated energy necessary to evaporate a neutral atom from the 'superheated' cluster anion, because vaporization may be a competing cooling mechanism.

\section{Experimental apparatus}

The experimental apparatus is described in detail elsewhere [17]. The cluster anions are produced directly by a laser vaporization source, where the internal vibrational temperature of the anions depends on various parameters of the source (seeding gas pressure, vaporization laser power, length and diameter of the extender, shape of the nozzle). The vibrational temperature of certain dimer anions can be determined from vibrationally resolved photoelectron spectra [5] and a vibrational temperature of about $200 \mathrm{~K}$ has been achieved under optimum cooling conditions.

After passing the skimmer, the negatively charged clusters are accelerated in a pulsed electric field. Depending on their times-of-flight the clusters separate into a chain of bunches with defined cluster sizes. The anion beam is directed into the source region of a 'magnetic bottle' time-of-flight electron spectrometer, and a selected bunch is irradiated by a laser pulse of a chosen photon energy. The detached electrons are guided by magnetic fields through a drift region towards an electron detector. The kinetic energy of the emitted electrons is related to their time-of-flight and the binding energy is the difference between the photon energy and the kinetic energy.

At kinetic energies higher than $0.2 \mathrm{eV}$ the transmission function is almost constant within $\pm 15 \%$. This has been verified by comparing certain refer- 
ence spectra (e.g. of $\mathrm{Cu}_{1}^{-}$) with the results obtained by other groups and by recording vibrationally resolved photoelectron spectra with a known intensity distribution of the single vibrational transitions (Franck-Condon distribution), especially for kinetic energies below $0.5 \mathrm{eV}$ [18]. In constrast to other types of electron spectrometers, the 'magnetic bottle' spectrometer suppresses any background signal from secondary electrons resulting from inelastic scattering of photoelectrons from surfaces close to the source region. The strong axial magnetic field acts as a shield of the source region.

In principle, a delayed emitted electron with a higher kinetic energy arrives at the electron detector at about the same time as one emitted immediately with a lower kinetic energy, so that the electron spectrum corresponds to a convolution of a time and an energy spectrum. However, the cluster anions remain only about $0.1 \mu \mathrm{s}$ in the source region of the spectrometer, and electrons emitted after this time are not detected. This time is about an order of magnitude shorter than the average time-of-flight of an electron with $E_{\text {kin }}<1 \mathrm{eV}$. We conclude that the measured photoelectron spectra mirror the kinetic energy distributions of those electrons emitted immediately and those with delays less than $0.1 \mu \mathrm{s}$.

Features corresponding to multiphoton processes can be identified by recording electron spectra at different laser fluxes. When a certain laser flux is exceeded, the relative intensity of the broad emission signal attributed to 'thermionic' emission increases with respect to the features assigned to direct photoemission, and the latter features show a significant broadening. Both observations can be explained if some of the cluster anions have absorbed and thermalized a photon before being struck by a second photon. At low laser fluxes both contributions to the photoelectron spectra vary linearly with the flux and the shape of the whole photoelectron spectrum does not change. Usually the flux is kept below this limit, assuring that each detected electron is emitted from a cluster which has interacted with a single photon.

\section{Results and discussion}

Fig. 1 displays photoelectron spectra of selected $\mathrm{W}_{n}^{-}$clusters with $n=1-10$ atoms recorded with a photon energy of $h \nu=4.025 \mathrm{eV}$ (XeCl excimer laser). The spectrum of the atom exhibits a multitude of sharp peaks, which can be assigned to transitions from the electronic ground state of $\mathrm{W}_{1}^{-}$to ground and excited electronic states of neutral $W_{1}$. The peak widths correspond to the energy resolution of the spectrometer. The relatively broad features observed in the spectrum of $\mathrm{W}_{2}^{-}$at lower binding energy (BE, between 1.5 and $2.5 \mathrm{eV}$ ) arise from additional vibrational and rotational degrees of freedom. A smooth signal is observed, increasing as BE increases ( $>3$ $\mathrm{eV})$. An overlap region composed of discrete and smooth features exists for BEs of 2.5-3.0 eV.

These two components are also observed in the spectra of larger clusters, with discrete features predominantly at lower BE (1.5-2.5 eV). We assign these features to direct photoemission from occupied $5 \mathrm{~d}-$ and $6 \mathrm{~s}$-derived valence orbitals of the clusters. The density of states in bulk $\mathrm{W}$ has a maximum close to the Fermi energy (due to the 5d bands) and decreases towards higher $\mathrm{BE}$ (dominated by the $6 \mathrm{~s}$ band) [19]. In a first approximation the photoelectron spectra resulting from direct photoemission reflect the distribution of the density of states in the cluster. The fine structure of these features depends strongly on the cluster size and develops gradually into the spectrum of the bulk as the size increases.

In all spectra at higher $\mathrm{BE}(>3 \mathrm{eV})$ only a few direct emission features can be identified. This region is dominated by a structureless, smooth emission signal, which increases monotonically towards higher BE. If the smooth emission signal can be attributed to 'thermionic' electron emission (TE), it should vary with the temperature of the cluster. Fig. 2 displays a comparison of two spectra of $W_{8}^{-}$ recorded at different laser fluxes. At high flux the TE signal is significantly larger due to a contribution from multiphoton processes.

Based on this assignment, we calculate Boltzmann distributions of the intensities $I_{\mathrm{TE}}$ of the TE electrons according to the function

$I_{\mathrm{TE}}=A \exp \left(-E_{\mathrm{kin}} / k_{\mathrm{B}} T\right)$,

and show the results in Fig. 1. The temperature $T$ corresponds to the photon energy $h \nu=4.025 \mathrm{eV}$ divided by the number of vibrational degrees of freedom of the cluster, and the initial temperature is neglected. This is reasonable for the small clusters 


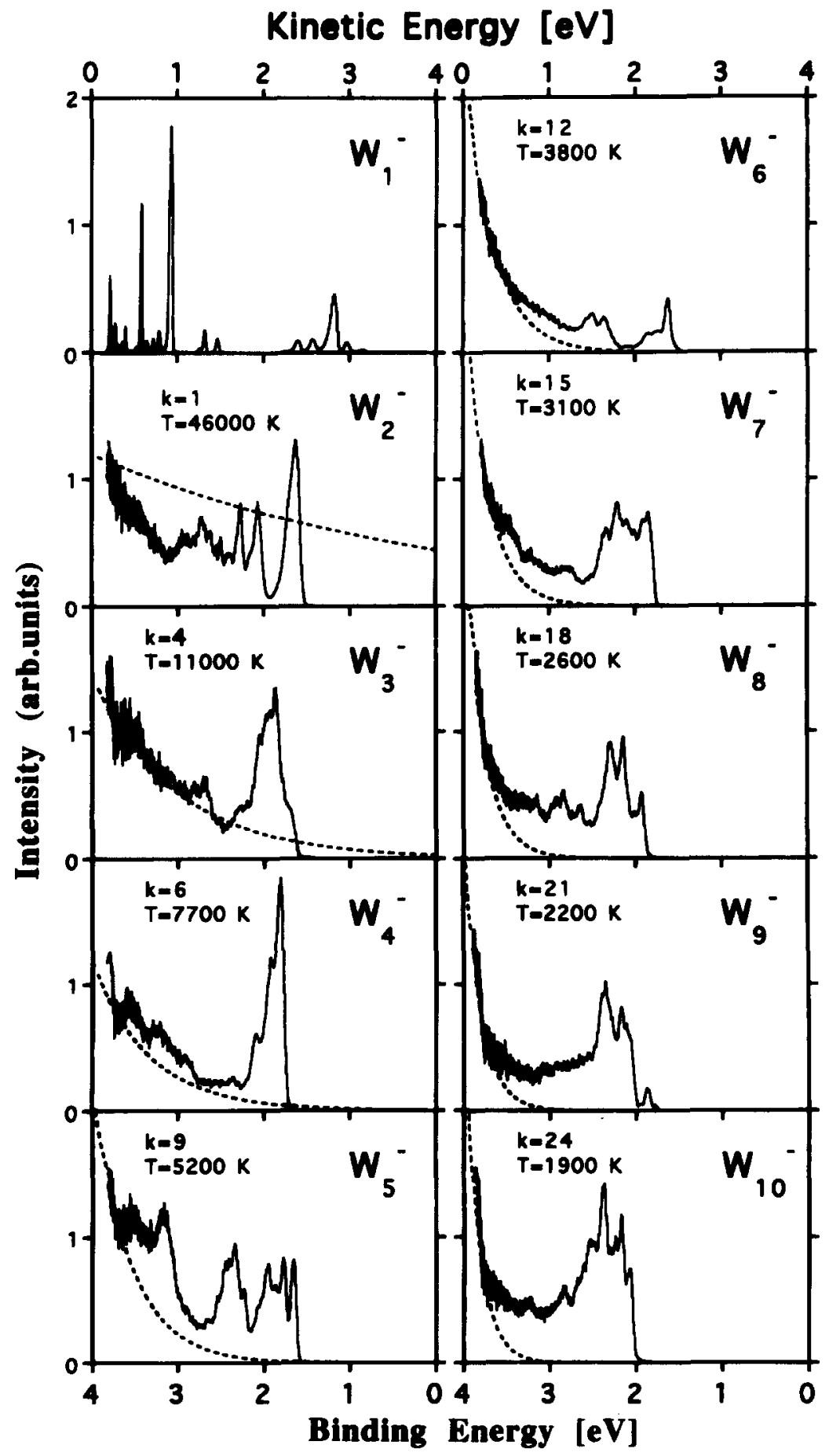

Fig. 1. Photoelectron spectra of $\mathrm{W}_{n}^{-}$clusters with $n=1-10$ atoms for a photon energy $h \nu=4.025 \mathrm{eV}$. The spectra are plotted versus the kinetic energy $E_{\text {kin }}$ (upper scale) and the binding energy BE (lower scale). The minimum kinetic energy is $0.2 \mathrm{eV}$. The laser flux is kept below $1 \mathrm{~mJ} / \mathrm{cm}^{-2}$ to avoid multiphoton processes. For each cluster the number of vibrational degrees of freedom $k$ and the temperature $T=4.025 \mathrm{eV} / k k_{\mathrm{B}}$ resulting from the absorption of a single photon are given. The dotted lines correspond to Boltzmann intensity distributions $I_{\mathrm{TE}}$ of the electrons emitted via TE calculated from the formula $I_{\mathrm{TE}}=A \exp \left(-E_{\mathrm{kin}} / k_{\mathrm{B}} T\right)\left(k_{\mathrm{B}}=\mathrm{Boltzmann}\right.$ constant). $A$ is adjusted to the experimental intensity. 


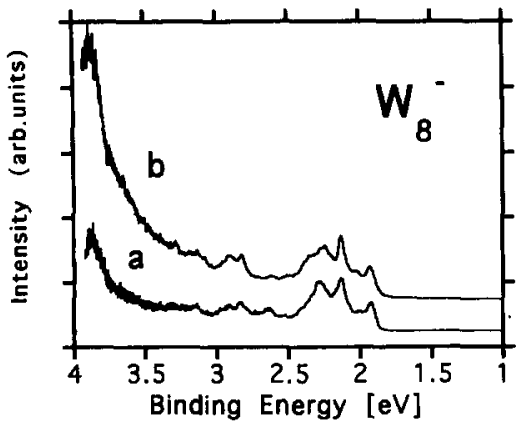

Fig. 2. Comparison of photoelectron spectra of $W_{8}^{-}$recorded at low $\left(1 \mathrm{~mJ} / \mathrm{cm}^{-2}\right)$ (a) and at moderate laser flux $\left(100 \mathrm{~mJ} / \mathrm{cm}^{-2}\right)$ (b). The photon energy is $h \nu=4.025 \mathrm{eV}$. At kinetic energies below $0.2 \mathrm{eV}$ the transmission of the electron spectrometer decreases resulting in a corresponding decrease in the electron intensity.

discussed here, since the temperatures resulting from the absorption of a $4 \mathrm{eV}$ photon are much higher (e.g. $3800 \mathrm{~K}$ for $\mathrm{W}_{6}^{-}$) than the estimated initial vibrational temperature $(\approx 200 \mathrm{~K})$. The factor $A$ is adjusted to fit the slope of the emission signal assigned to thermionic emission.

The simple exponential distributions agree surprisingly well with most of the data. The temperature of the clusters induced by photoabsorption decreases with increasing cluster size, since the photon energy is distributed over an increasing number of vibrational degrees of freedom and the average kinetic energy of the TE electrons is smaller. For $\mathrm{W}_{10}^{-}$, the TE signal shows up as a steep increase in the intensity between 3.7 and $3.8 \mathrm{eV}\left(0.2-0.3 \mathrm{eV} E_{\mathrm{kin}}\right)$.

The signal assigned to TE is observed in the dimer. Although the simple fit disagrees completely in this case, it reproduces the spectra of the trimer and tetramer relatively well. With increasing cluster size the TE signal exhibits some fine structure (e.g. for $\mathrm{W}_{4}^{-}$), but it is not clear whether this is caused by a superposition of direct photoemission peaks or by a non-thermal distribution of the TE electrons. For $\mathrm{W}_{6}^{-}$the TE signal agrees well with the Boltzmann distribution.

With increasing cluster size $(n>9)$ the TE signal is confined to the lowest kinetic energies $\left(E_{\mathrm{kin}}<0.3\right.$ $\mathrm{eV}$, upper scale in Fig. 1) and with further increasing cluster size the kinetic energy of the TE electrons is too low to be detected with our spectrometer. In the spectra of the larger clusters (e.g., $W_{8}^{-}-W_{10}^{-}$) a smooth, almost constant emission signal is observed between the features assigned to direct emission and TE. This signal may arise from the electron-'phonon' scattering process, such as observed in $\mathrm{C}_{60}^{-}$[15], where an outgoing electron loses energy due to the vibrations of the molecule. Inelastic electron-phonon scattering is common in the photoelectron spectra of bulk materials and the cross section depends on the density of vibrational states. This is consistent with the increasing intensity of this emission signal with increasing cluster size.

The description of the internal energy of the clusters by a temperature is only approximate, since the individual clusters of an ensemble have absorbed exactly the same amount of energy $(=4.025 \mathrm{eV})$. This results in an upper limit of the kinetic energy of the electrons emitted via TE $(=4.025 \mathrm{eV}$ minus the electron affinity), which cannot be described by a Boltzmann function [16].

The TE signal and the direct emission features have similar intensities in the data displayed in Fig. 1. The probability for a photon interacting with a cluster to detach an electron directly or to 'heat' the cluster is approximately equal, even at a photon energy significantly higher than the electron affinity. Therefore, direct photoemission is not the dominant process governing the interaction of a photon (with energy above threshold) and a cluster.

The integral intensity of the thermionic emission appears to decrease with increasing cluster size (Fig. 1), possibly due to the increasing timescale of the delayed emission. An increasing number of TE electrons are emitted with a delay larger than $0.1 \mu \mathrm{s}$ and will therefore not be detected. As the number of degrees of freedom increases, the photon energy is distributed over a larger number of vibrational modes, and it takes longer to focus the energy back into the electron emission process. If the time constant of the emission is much longer than the timescale of the experiment $(0.1 \mu \mathrm{s})$, only direct emission features would be observed in the spectra.

Thermionic emission is only one possible cooling mechanism for a 'superheated' cluster. A fast and effective cooling process is evaporation according to

$$
\begin{aligned}
\mathbf{M}_{n}^{-}+h \nu & \rightarrow\left(\mathbf{M}_{n}^{-*}\right)_{E_{1}} \\
& \rightarrow\left(\mathbf{M}_{n-1}^{-*}\right)_{E_{2}}+\mathbf{M}_{1} \\
& \rightarrow\left(\mathrm{M}_{n-2}^{-*}\right)_{E_{3}}+\mathbf{M}_{2} \\
& \rightarrow \ldots,
\end{aligned}
$$


where no electron is emitted. If fragmentation is the preferred decay channel, no TE signal will appear in the photoelectron spectrum. The branching ratio of the two cooling mechanism depends on the rate constants, and the faster process dominates. The time constant depends exponentially on the energy threshold, which is the binding energy of the outermost electron (ionization potential, electron affinity) in the case of TE, and the binding energy of the atom or dimer in the case of evaporative cooling. TE is mostly observed for neutral clusters of the refractory metals ( $\mathrm{W}, \mathrm{Nb}, \mathrm{Ta}, \ldots)$ and carbon. For these materials the threshold for evaporation is higher than the ionization potential.

The vertical detachment energies (VDEs) of the $\mathrm{W}_{n}^{-}$clusters can be extracted directly from the photoelectron spectra (Table 1). The VDEs correspond roughly to the electron affinities of the clusters [20]. Due to the lack of experimental data on the dissociation energies we have used a model proposed by Miedema [21] for the neutral clusters. The energy necessary for the evaporation of a single atom $\mathrm{D}_{n}$ from a neutral cluster with $n$ atoms is estimated based on the heat of evaporation of the solid, $\Delta H_{\text {vap }}$ (8.81 eV [22]), and a correction for the large fraction of surface atoms in a cluster. The surface atoms have a smaller binding energy due to the smaller number of nearest neighbours and therefore $\mathrm{D}_{n}$ increases with increasing $n$ approaching $\Delta H_{\text {vap }}$. This model may be applied to clusters with highly localized

Table 1

Vertical detachment energies $( \pm 0.05 \mathrm{eV})$ for tungsten clusters. The electron affinity of the atom is $0.815(8) \mathrm{eV}$ [23]

\begin{tabular}{rlll}
\hline$n$ & EA (eV) & $n$ & EA (eV) \\
\hline 2 & 1.46 & 12 & 1.96 \\
3 & 1.44 & 13 & 1.89 \\
4 & 1.64 & 14 & 1.99 \\
5 & 1.58 & 15 & 2.21 \\
6 & 1.48 & 16 & 2.31 \\
7 & 1.72 & 17 & 2.40 \\
8 & 1.74 & 18 & 2.44 \\
9 & 1.73 & 19 & 2.41 \\
10 & 1.94 & 20 & 2.44 \\
11 & 1.95 & 21 & 2.52 \\
\hline
\end{tabular}

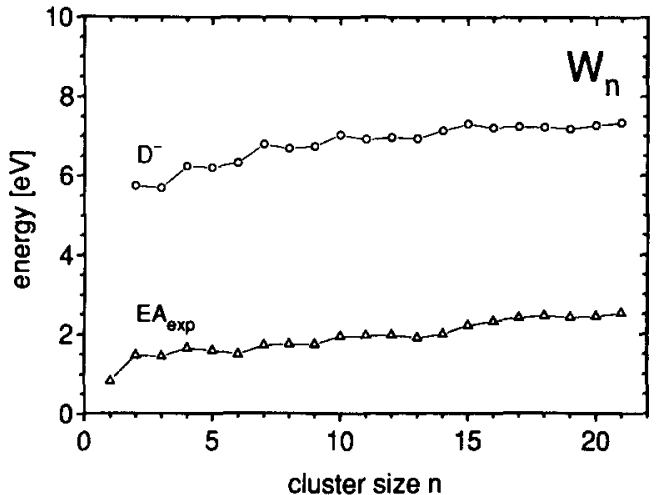

Fig. 3. Experimental vertical detachment energies for tungsten clusters with an uncertainty of $\pm 0.05 \mathrm{eV}$ compared to the dissociation energies $\mathrm{D}_{n}^{-}$corresponding to the reaction $\mathrm{W}_{n}^{-} \rightarrow \mathrm{W}_{n-1}^{-}+$ $W$. The dissociation energies of the cluster anions are estimated using a model proposed by Miedema [21] together with the electron affinities using a Born-Haber cycle.

bonds such as van der Waals clusters or to transition metal clusters with a main contribution from localized $\mathrm{d}$ electrons to the binding.

The dissociation energy of a negatively charged cluster $\mathrm{D}_{n}^{-}$can be calculated from the electron affinities using a Born-Haber cycle

$\mathrm{D}_{n}^{-}=\mathrm{D}_{n}+\mathrm{EA}(n)-\mathrm{EA}(n-1)$.

Fig. 3 displays a comparison of these energies for the $\mathrm{W}_{n}^{-}$clusters with $n=2-21$. The energies for evaporation of an atom are approximately 3-4 times larger than the VDEs for the $\mathrm{W}_{n}^{-}$clusters. The neglect of evaporative cooling is consistent with the intense TE signal observed in the photoelectron spectra (Fig. 1). For clusters of other materials the ratio between the two energies is different. For clusters with a low dissociation energy and a high electron affinity, in particular, evaporative cooling may be the preferred decay channel, and no TE signal would be observed. We plan to study various clusters of different materials and compare the intensities of the TE signal with the electron affinities and energies necessary for the evaporation of an atom.

\section{Conclusion}

Thermionic emission has been identified as a fundamental process competing with the direct pho- 
toemission from vibrationally 'cold' cluster anions following the absorption of a single photon above threshold. The kinetic energy distributions of the electrons emitted via thermionic emission from single-photon events have been studied systematically. They can be described qualitatively by a Boltzmann function with the temperature calculated from the photon energy divided by the number of vibrational degrees of freedom. Thermionic emission following single-photon absorption can be observed only for small clusters, because the timescale of the process increases rapidly beyond the timescale of the experiment with an increasing number of degrees of freedom. Thermionic emission seems to be a major cooling channel for cluster anions, as long as the electron affinity is smaller than the dissociation energy and fast relaxation of the electronic excitation (thermalization) occurs.

\section{Acknowledgement}

We thank P.S. Bechthold for the use of his laser vaporization source for the generation of the tungsten clusters. B. Kessler and R.O. Jones are gratefully acknowledged for lively and encouraging discussions. This project was supported in part by the Deutsche Forschungsgemeinschaft.

\section{References}

[1] C. Herring and M.H. Nichols, Rev. Mod. Phys. 21 (1949) 185.

[2] N.W. Ashcroft and N.D. Mermin, Solid state physics (international edition) (Saunders, London, 1976) ch. 18.
[3] J.L. Persson and R.L. Whetten, Chem. Phys. Letters 147 (1988) 168.

[4] P.J. Hay, J. Chem. Phys. 76 (1982) 502.

[5] G. Ganteför, D.M. Cox and A. Kaldor, J. Chem. Phys. 94 (1991) 854, and references therein.

[6] P.D. Dao and A.W. Castleman, J. Chem. Phys. 84 (1986) 1435.

[7] T. Leisner, K. Athanassenas, O. Echt, O. Kandler, D. Kreisle and E. Recknagel, Z. Physik D 20 (1991) 127.

[8] A. Amrein, R. Simpson and P. Hackett, J. Chem. Phys. 94 (1991) 4663.

[9] T. Leisner, K. Athanassenas, D. Kreisle, E. Recknagel and O. Echt, J. Chem. Phys. 99 (1993) 9670.

[10] H. Weidele, St. Becker, H.-J. Kluge, M. Lindinger, L. Schweikhard, C. Walther, J. Ziegler and D. Kreisle, Surface Rev. Letters, in press.

[11] E.E.B. Campbell, G. Ulmer and I.V. Hertel, Phys. Rev. Letters 67 (1991) 1986.

[12] K.R. Lykke and P. Wurz, J. Phys. Chem. 96 (1992) 3191, 10129.

[13] Y. Zhang and M. Stuke, Phys. Rev. Letters 70 (1993) 3231

[14] D. Ding, J. Huang, R.N. Compton, C.E. Klots and R.E. Haufler, Phys. Rev. Letters 73 (1994) 1084.

[15] L.-S. Wang, J. Conceicao, C. Jin and R.E. Smalley, Chem. Phys. Letters 182 (1991) 5.

[16] D. Ding, R.N. Compton, R.E. Haufler and C.E. Klots, J. Phys. Chem. 97 (1993) 2500.

[17] C.-Y. Cha, G. Ganteför, and W. Eberhardt, Rev. Sci. Instrum. 63 (1992) 5661.

[18] H. Handschuh, G. Ganteför, and W. Eberhardt, submitted for publication.

[19] M. Posternak, H. Krakauer, A.J. Freeman and D.D. Koelling, Phys. Rev. B 21 (1980) 5601.

[20] C.-Y. Cha, G. Ganteför and W. Eberhardt, J. Chem. Phys. 100 (1994) 1.

[21] A.R. Miedema, Z. Metallkunde 69 (1978) 287; Faraday Symp. Chem. Soc. 14 (1980) 136.

[22] M.W. Chase Jr., C.A. Davies, J.R. Downey Jr,, D.J. Frurip, R.A. McDonald and A.N. Syverud, J. Phys. Chem. Ref. Data 14 (1985) No. 1.

[23] H. Hotop and W.C. Lineberger, J. Phys. Chem. Ref. Data 14 (1985) 731. 